\title{
TeaMPI-Replication-Based Resilience Without the (Performance) Pain
}

\author{
Philipp Samfass ${ }^{1(\bowtie)}$, Tobias Weinzierl ${ }^{2}$, Benjamin Hazelwood ${ }^{2}$, \\ and Michael Bader ${ }^{1}$ \\ 1 Technische Universität München, 85748 Garching, Germany \\ \{samfass, bader\}@in.tum.de \\ 2 Computer Science, Institute for Data Science, Durham University, \\ DH13LE Durham, Great Britain \\ tobias.weinzierl@durham.ac.uk, ben.hazelwood@featurespace.co.uk
}

\begin{abstract}
In an era where we can not afford to checkpoint frequently, replication is a generic way forward to construct numerical simulations that can continue to run even if hardware parts fail. Yet, replication often is not employed on larger scales, as naïvely mirroring a computation once effectively halves the machine size, and as keeping replicated simulations consistent with each other is not trivial. We demonstrate for the ExaHyPE engine - a task-based solver for hyperbolic equation systemsthat it is possible to realise resiliency without major code changes on the user side, while we introduce a novel algorithmic idea where replication reduces the time-to-solution. The redundant CPU cycles are not burned "for nothing". Our work employs a weakly consistent data model where replicas run independently yet inform each other through heartbeat messages whether they are still up and running. Our key performance idea is to let the tasks of the replicated simulations share some of their outcomes, while we shuffle the actual task execution order per replica. This way, replicated ranks can skip some local computations and automatically start to synchronise with each other. Our experiments with a production-level seismic wave-equation solver provide evidence that this novel concept has the potential to make replication affordable for large-scale simulations in high-performance computing.
\end{abstract}

\section{Introduction}

Supercomputing roadmaps predict that machines soon will suffer from hardware unreliability [11]. A linear correlation between system size and the number of failures has already been observed [27], as effects alike bias temperature instabilities or hot carrier injection diminish the mean time between failures (MTBF) for the individual components. For the next generation of machine sizes, a preserved or reduced MTBF however implies that codes have to be prepared for parts of the machine going down unexpectedly, either through hard errors or soft errors corrupting the code's state. Alternatively, parts might become unacceptably slow as hardware or software error correction [22] step in. We thus need

(C) Springer Nature Switzerland AG 2020

P. Sadayappan et al. (Eds.): ISC High Performance 2020, LNCS 12151, pp. 455-473, 2020.

https://doi.org/10.1007/978-3-030-50743-5_23 
resilient codes. Numerical simulations will have to be at the forefront here. With their massive concurrency going full speed and their strong causal dependencies between intermediate results they are vulnerable to hardware failures.

For numerical simulations, we distinguish three strategies to inject resilience: (i) Codes can be prepared algorithmically to recover from drop-outs of compute nodes. (ii) Codes can checkpoint and restart if hardware fails. (iii) Codes can run computations redundantly.

The first variant works only if the underlying problem allows us to recover information even if data is "lost". Elliptic equations fall into this category: If we know the solution around a region that has dropped out, we can reconstruct the solution within the domain $[1,17]$. Another example for algorithmic recovery is the combination technique, where a drop-out of some data might (slightly) reduce the solution accuracy but the overall algorithm can cope with it [18]. In both cases, the numerical scheme itself has to be resiliency-ready.

Checkpointing works more in a black-box fashion, but the time to write a checkpoint has to be significantly smaller than the MTBF. We also have to be willing to spend CPU cycles and energy on I/O, which typically is costly [15]. For in-memory checkpointing which mitigates the speed and energy penalty, we need "spare" storage. As checkpoints are costly one way or the other, partial checkpoint-restart is a must. Containment domains [9] for example ask the programmer to decompose the application into task-similar constructs with manual state preservation, error detection and recovery. Some tasking runtime systems such as ParSEC [4] provide a framework for the "automatic" re-execution of task sub-graphs in combination with checkpointing. A sophisticated example for checkpointing is to run the recalculation with a different numerical scheme [22]. This realises a hybrid between an algorithmic approach and checkpoints.

If algorithmic resiliency is not at hand and checkpointing cannot be afforded, replication of work, i.e., data redundancy, is the prime solution. If a node or memory drops out, we simply swap in the replicated data. Cloud computing, sensor networks, desktop grids, peer-to-peer networks, and almost every other field that requires resilient computations [5] base their fault tolerance upon the idea of replicating resources. Capability high-performance computing (HPC) in contrast tends not to use replication. If we duplicate a computation, we effectively half the machine - which renders the prime character of capability computing absurd. Since supercomputers however tend to become so ill-balanced w.r.t. I/O capabilities vs. compute resources that we cannot afford to checkpoint frequently, we will eventually be forced to employ replication nevertheless $[12,13,24]$. We therefore need to reduce its pain.

Our paper introduces a novel idea to do so, together with a prototypical implementation of team-based MPI replication, called teaMPI. We demonstrate its potential for a high-order discontinuous Galerkin code for hyperbolic equation systems, i.e., a solver for which we are not aware of any straightforward algorithmic resiliency strategy. Our approach relies on replication on the MPI rank level. Each rank is replicated $K$ times, while the simulation per rank is phrased in tasks. A task is an atomic unit, i.e., it has a well-defined input and 
output and, once it becomes ready, can be executed without any further dependencies. To benefit from our techniques, codes need not be task-based only, but the heavy workload should be phrased as tasks. Furthermore, we require that tasks allow us to send their outcome via MPI, and they should have some slack, i.e., should not be part of the critical path. That is, there is some freedom to move their startup time around, without immediately penalising the overall time-to-solution.

With such tasks, we can replicate each rank $K$ times without a $K \times$ overhead in compute time: We shuffle the task execution order per replication, i.e., we make each rank process sets of ready tasks in a slightly different order. Furthermore, we let each rank offer its task outcomes to other replicas. Whenever a task is about to be executed on a rank, this rank now can first check whether the task outcome is already available from a replica. If so, it skips the execution. All techniques affect the task runtime, i.e., can be invisible to the simulation [29]. To the best of our knowledge, this is the first approach offering full simulation replication without a full multiplication of compute workload. It is thus a fundamental contribution towards affordable replication-based resiliency in HPC.

While our paper focuses on speed of execution, we can detect certain hard failures as well, using a concept called heartbeats. Since we keep redundant copies of data, we could, similar to RAID systems, replace corrupt ranks. However, a discussion and presentation of such a swapping strategy is beyond the scope of this paper. Furthermore, we do not yet link our work to MPI-based run-through-stabilisation techniques $[3,8,14]$, which inject further technical and implementation difficulties. Finally, replication in HPC remains a double-edged sword: While it offers fault-tolerance, it also requires to use more memory, network bandwidth and compute units, i.e., CPU hours, per simulation run. Our approach reduces the compute cost compared to naïve replication. We however neglect the increased memory [2] and network stress. For many applications, users will have to balance the replication-based resilience against these facets of increased cost.

The remainder of the text is organised as follows: We establish our terminology in Sect. 2 and sketch the replication mechanisms. Our core contribution is the introduction of the task-based result sharing (Sect.3) which eventually reduces the workload per rank whenever results from a replica drop in on time. The realisation and encapsulation of the whole idea is subject of discussion in Sect. 4, before we study the method's runtime implications (Sect.5). A brief conclusion and outlook in Sect. 6 wrap up the discussion.

\section{Team-Based Resiliency with Heartbeats}

We first introduce the terminology which underlies our algorithmic contributions. The terminology is also adopted by our software teaMPI which realises the proposed ideas. teaMPI plugs into the MPI standard profiling interface (PMPI). By mapping physical ranks onto logical numbers and altering the MPI communicator size (number of ranks) exposed to SPMD user code, it transparently reorganises and replicates MPI ranks in multiple teams: 


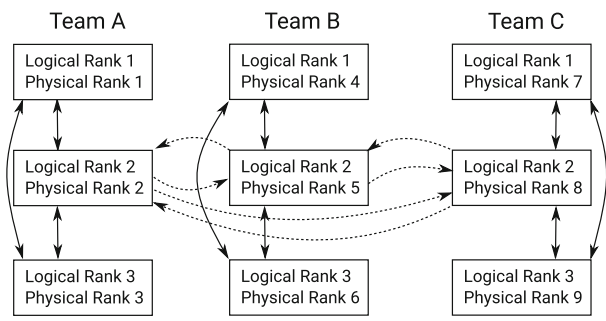

Fig. 1. Illustration of a replication-based run with three teams, each hosting three ranks. In the baseline code, ranks communicate only with their team members (solid arrows), but they send heartbeats in regular time intervals to their replicas (dotted arrows, only illustrated for the logical ranks 2).

Definition 1 (Team). All the ranks of an application (without any redundancy) form a team. If we run a code with $K$-fold redundancy, the global set of ranks is split into $K$ teams. Each team consists of the same number of ranks, sees only the ranks of its own team, and runs asynchronously from all the other teams. Each team consequently hosts one application instance of its own.

With this definition, each rank belongs uniquely to one team. If there are $K$ teams, each rank has $K-1$ replicas belonging to other teams. The teams are completely autonomous, i.e., independent of the other teams, and therefore consistent only in themselves: We run the code $K$ times and each run completes all computations and has all data. There is neither some kind of lockstepping nor any data sharing in this baseline version of teaMPI.

With teaMPI, our team-based replication for SPMD is totally transparent: An application neither does need to replicate data structures nor does it need to be aware of the replication. Teams are formed from subsets of MPI ranks at the simulation start-up. All subsequent communication calls to both point-to-point and collective MPI routines are mapped by teaMPI to communication within the teams. We work with both data and computation redundancy: Each team is a complete application instance, and, due to SPMD, a send within team A from rank $r_{1}^{(A)}$ to rank $r_{2}^{(A)}$ will have a matching send in team B from $r_{1}^{(B)}$ to $r_{2}^{(B)}$.

Running an application with teaMPI logically means the same as using a communicator decomposition and making each communicator run the whole simulation. Teams do not have to be consistent all the time. They are weakly consistent and fully asynchronous without added overhead for message consistency checking. Instead of an in-built sanity check for MPI messages, we rely on low-frequency consistency checks:

Definition 2 (Heartbeat). Each rank in each team issues a heartbeat after every $\Delta t_{H B}$ seconds. Heartbeats are sent to all replica of a rank (replication multicast) and only carry the elapsed wall time since the last heartbeat. They are sent out in a fire-and-forget fashion. 
While all other MPI communication is restricted to intra-team only and transfers data from the user space, heartbeats are simple non-blocking messages exchanged between the replicas which do not carry any user data. We clearly distinguish intra-team from inter-replica communication (Fig. 1). The latter is hidden from the user code and is no arbitrary inter-team data exchange. It only "couples" replicas. As mainstream HPC machines lack support for hard real-time scheduling, we weaken the $\Delta t_{\mathrm{HB}}$ property: We launch a task which reschedules itself all the time with a low priority and issues a heartbeat as soon as at least $\Delta t_{\mathrm{HB}}$ seconds have expired since the last heartbeat task.

Definition 3 (Team divergence). A rank of a team diverges if the time inbetween two heartbeats increases compared to this in-between time in other teams. A team diverges if at least one rank of this team diverges.

Divergence is a relative quantity. It results from a comparison of local in-between times to the time stamps carried by arriving messages. If a rank is overbooked with tasks and its heartbeat task thus is issued with $\Delta t \gg \Delta t_{\mathrm{HB}}$, it is reasonable to assume that any replica faces a similar delay of heartbeats as it "suffers" from high workload, too. Divergence is also an observable property: A rank can identify a slowing down replica, and a slow rank can identify itself as diverging by receiving faster heartbeats from other ranks. Finally, divergence is an asynchronous property, as we use the timestamps written into the heartbeats to compute in-between times. Local clocks do not have to be synchronised and we try to eliminate MPI message delivery/progression effects.

Divergence nevertheless remains a statistic quantity: As we work in a multitasking MPI+X environment, a single late heartbeat is not a reliable indicator that a rank is suffering from errors or overheating and thus is going down. If we observe divergence over a longer time span or define well-suited timeouts $\Delta t_{\mathrm{HB}} \geq \Delta t_{\text {timeout }}$, we can however spot failing ranks.

Each team does exactly the same calculations and thus eventually reaches the same state, but the heartbeats run asynchronously on-top and do not impose any synchronisation. They can identify that a rank is going down as it becomes slower, or they can identify complete drop-outs. They are ill-suited to spot data inconsistency. However, we hypothesize that data inconsistencies eventually manifest in corrupted data and thus in the drop-out of a complete team; which we can detect again.

Our transparent replication is similar to the one used in RedMPI, e.g. [16]. RedMPI and other replication models however enforce a strong consistency model among replicated ranks, i.e., make all replicas have exactly the same state subject to temporal shifts. Individual MPI messages are double-checked against replicas for soft errors. This adds synchronisation. Strong consistency on the message level furthermore becomes particularly challenging when wildcard MPI receive operations are used. We avoid this cliff.

To constrain overhead, many approaches do not replicate data and computations automatically and persistently, but enable replication on-demand. The ARRIA distributed runtime system, e.g. [28] schedules and replicates tasks based 


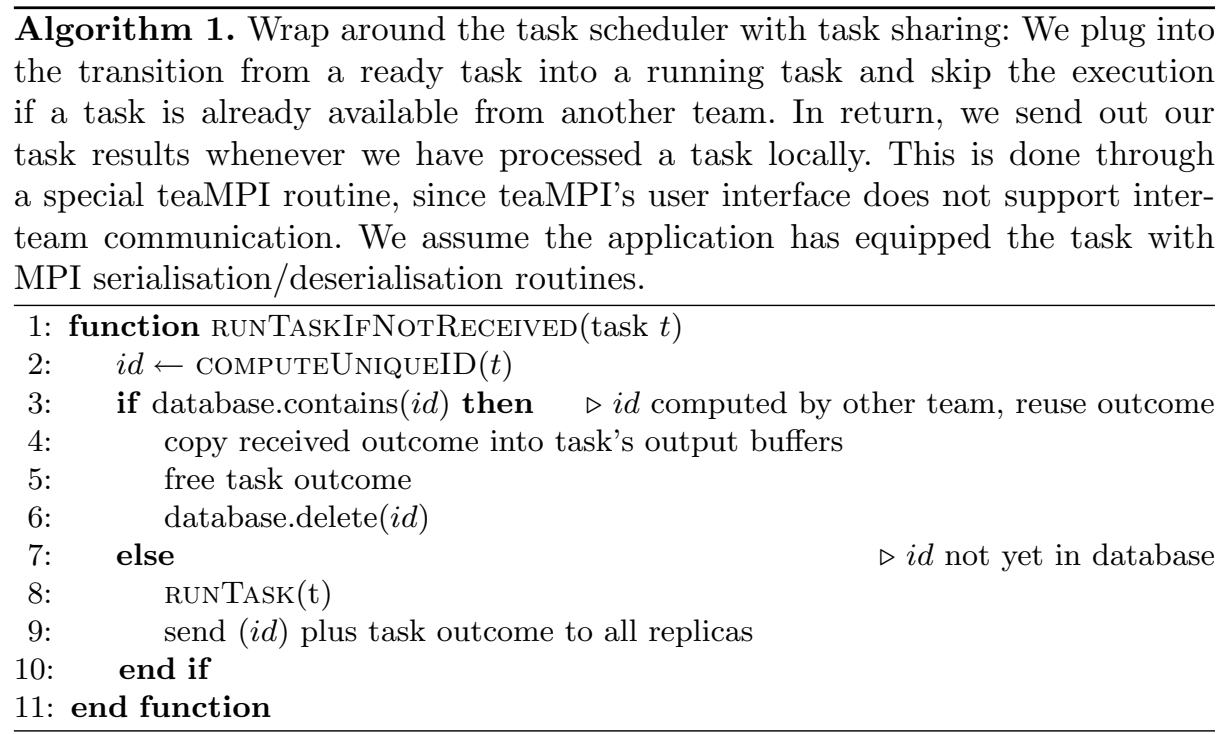

on predictions about their probability of failure, while the work in [23] allows to spawn resilient tasks that use either replicated execution or checkpointing for resilience depending on the programmer's choice. Our replication is persistent.

\section{Task Sharing}

teaMPI makes both data as well as all computations redundant. In order to save compute time, we however propose an algorithm where teams exchange outcomes of tasks.

Definition 4 (Shareable task). The tasks of interest for this paper have four important properties: (i) They are compute-heavy, i.e., exhibit a high arithmetic intensity. (ii) They are not a member of the critical path. We can delay their execution once they are spawned by some time without slowing down the application immediately. (iii) They have an outcome with a relatively small footprint relative to their compute cost, and the outcome is serialisable. We can send it around through MPI. (iv) They have a globally unique id.

Uniqueness incorporates both the data the task is working on plus its action. As we work with a time stepping solver, each task also is unique by the time step it belongs to. Two tasks $t^{(A)}$ and $t^{(B)}$ from two different teams $A$ and $B$ thus have the same id if and only if they perform the same action on the same data of their respective application and are issued by the same time step.

From a user's point of view, teaMPI allows ranks only to exchange data within their team, while teaMPI itself exchanges heartbeats between teams. To reduce the total cost despite the replication, we introduce further inter-team 


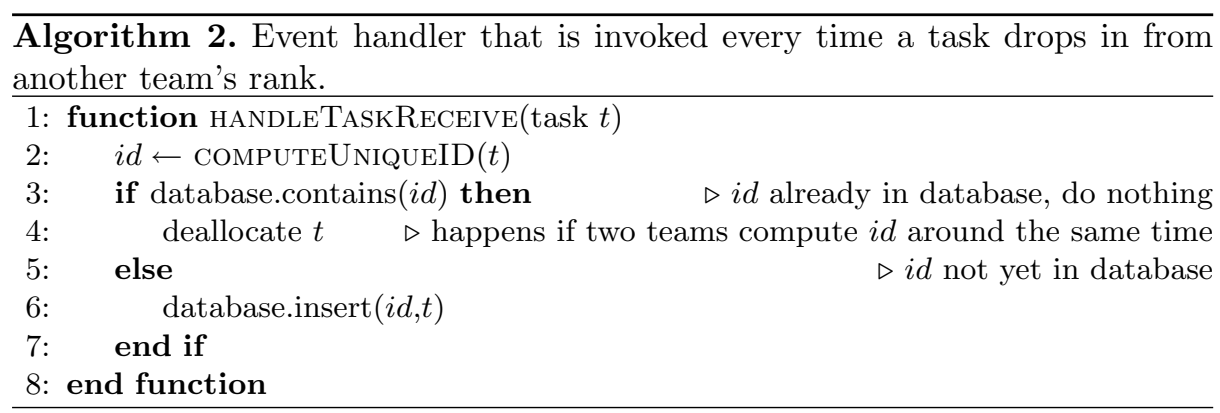

data flow from hereon. Both this further flow and the heartbeats however do not introduce arbitrary point-to-point connections. They solely remain inter-replica.

Our extension is a straightforward augmentation of the task runtime (Algorithm 1): The runtime on a rank sends the outcome of any shareable task that it has completed to all replicas, i.e., all the corresponding ranks in the other teams. They receive and store them in a database. For every ready shareable task that is to be launched, we hence validate first that its outcome has not yet been received. If this is not the case, we execute the actual task (and eventually distribute its result). If a task outcome is already in our database, we do not have to compute the task any more. It is sufficient to roll over the received task result and to skip the actual computation. To make this work, the scheduling is complemented by a receive handler listening for task results (Algorithm 2).

The database is a map from task ids onto task outcomes. An entry in the database indicates that a task outcome has been received. A database of received tasks as sketched so far would grow monotonically, since tasks might drop in while they are computed. We thus equip each database entry with a timeout and run a garbage collection regularly. It removes all entries and cleans up buffers for tasks which are considered to be too old. Such a timeout could rely on heartbeat counts ("received more than $\mathrm{x}$ heartbeats before"). For explicit timestepping, it is however more convenient to use the time step counter. Entries older than the most recent time step won't be used anymore and can safely be discarded.

The algorithmic blueprint so far saves compute cost whenever a team lags behind. The team running ahead completes its tasks and sends out the results. The team behind picks up the results and skips its own computations. If two teams are roughly running in-sync, we have to modify the scheduling slightly to benefit from the exchange between replicas:

Definition 5 (Task shuffling). Let $\left\{t_{1}, t_{2}, t_{3}, \ldots\right\}$ be a set of tasks that are issued as ready or released as ready in one rush by our application. The first team $A$ schedules $\left\{t_{1}^{(A)}, t_{K+1}^{(A)}, t_{2 K+1}^{(A)}, \ldots\right\}$ prior to $\left\{t_{2}^{(A)}, t_{K+2}^{(A)}, t_{2 K+2}^{(A)}, \ldots\right\}$ and so forth. The second team $B$ schedules $\left\{t_{2}^{(B)}, t_{K+2}^{(B)}, t_{2 K+2}^{(B)}, \ldots\right\}$ prior to $\left\{t_{3}^{(B)}, t_{K+3}^{(B)}, t_{2 K+3}^{(B)}, \ldots\right\}$ and eventually $\left\{t_{1}^{(B)}, t_{K+1}^{(B)}, t_{2 K+1}^{(B)}, \ldots\right\}$. This pattern continues for all teams. 
Each team permutes its shareable tasks modulo the number of teams. In practice, it is convenient to realise this through task priorities where high priority tasks are scheduled prior to low priority tasks. We start from the application's task priorities but then add subpriorities with a modulo counter which realise the shuffling. Such shuffling even works for applications which do not issue tasks in a batch but fire them one by one. Shuffling weakens the task scheduling consistency, and effectively the data consistency between the teams. The only situation where it might not ensure a differing task execution ordering is when ranks issue tasks non-deterministically. In this case, the randomness plus the shuffling might yield similar task execution orders for different teams. Yet, this is unlikely.

\section{Implementation}

teaMPI is implemented as a $\mathrm{C}++$ library. Using the PMPI interface, teaMPI intercepts the relevant MPI calls and redirects them onto communicators or different physical ranks, respectively. We mainly wrap blocking and nonblocking point-to-point routines as well as collectives. Relying on PMPI makes teaMPI portable. For a replication factor $K$, an application with $R$ ranks is started with a total of $K \cdot R$ ranks. This yields $K$ teams with $R$ ranks each. Within MPI's initialisation, teaMPI creates subcommunicators for all intra-team communication. Each subsequent user MPI call is hijacked by teaMPI and internally mapped onto an MPI call on the appropriate subcommunicator.

\subsection{Implementation Decisions}

Heartbeats Without a Hard Real-Time Environment. Issuing heartbeats during the simulation requires particular care. If we make heartbeats dependent on the progression of the numerical simulation (for example by posting a heartbeat after every time step), a single slow rank would delay the heartbeats of other ranks in its team: In classical domain-decomposition approaches (as in our example application), point-to-point messages to "neighbour" ranks are required before a new simulation time step can be started. A single slow rank will therefore delay its neighbours, too. With a heartbeat after each time step, it would thus not be possible to isolate an individual slow or failing rank. We could only identify teams hosting a slow or dropped-out rank.

teaMPI's heartbeats are issued by a special heartbeat task on each rank. The heartbeat reschedules itself until program termination. It stores the time stamp of the most recent heartbeat. Whenever invoked, the heartbeat task checks whether at least $\Delta t_{\mathrm{HB}}$ seconds have elapsed since the last heartbeat. If so, a new heartbeat message is issued and the stored time stamp is updated. We rely on two assumptions: (i) Tasks run agnostic of MPI synchronization and the progression of the numerical algorithm. That is, even if some ranks cannot proceed with their next time step due to missing MPI messages, they will nevertheless process the heartbeat task. (ii) If a rank crashes, it stops issuing heartbeats. If it slows 
down significantly, also its heartbeat task will be triggered less often, resulting in increased time intervals between two heartbeats. This allows us to single out a failing rank. The $\Delta t_{\mathrm{HB}}$ ensures that the system is not flooded with heartbeat messages and is not overly sensitive to small performance fluctuations [6].

In our implementation, we use Intel's Threading Building Blocks (TBB), an abstraction from the actual hardware threading. TBB lacks support for real-time tasking. This introduces uncertainty. We do not know when exactly heartbeats are triggered. We can not ensure that the time in-between two heartbeat sends equals the prescribed $\Delta t_{\mathrm{HB}}$. We might even end up with situations where a rank $r^{(A)}$ sends more heartbeats to its replica $r^{(B)}$ than the other way around.

This challenge seems to be amplified when ranks deploy their results to replicas. Any deployment moves computational load between replicas and thus, on purpose, unbalances ranks belonging to different teams. Real-time heartbeats would be agnostic of this. However, their usage would contradict our assumption that hardware failures announce themselves often through a performance degradation. We launch heartbeats with a fixed, reasonably high priority and rely on the runtime to schedule the heartbeats fairly and, more importantly, roughly with the same time intervals $\Delta t_{\mathrm{HB}}$ on all teams. It is obvious that a more mature solution would use a burn-in phase without any replication data sharing to determine a proper priority. The important implementation remark is that we use a comparison of local heartbeat in-between times to the in-between times of received heartbeats to identify slow downs.

On the receiver side, we rely on MPI polling for the heartbeats: Whenever a heartbeat task becomes active, we both send out our heartbeat (multicast) and probe on available incoming ones. If there are heartbeats in the MPI queue, we dump them into a local array. Unexpected message arrivals should be avoided in MPI. Yet, heartbeats do not induce a major runtime penalty. With only the timestamp, their message footprint is small.

Task Sharing. For the inter-team data exchange, teaMPI does not hijack standardised MPI, but offers dedicated routines. These routines expose additional inter-team communicators and teaMPI's knowledge about the number of teams to the application. This way, the application can circumvent native MPI communication which is wrapped by teaMPI and only designed for intra-team exchange. Task outcome sharing is multicasts sending one piece of data to all replicas. To use these routines, we make use of a previously developed communication infrastructure that relaxes the binding of tasks to their spawning rank [26]: tasks and their outcomes can migrate dynamically at runtime to other processes. For this, we add meta data (the unique ids) to the tasks and MPI sending/receiving wrappers around both the meta data, input arguments and output. All the wrapping is not for free: Task outcome sharing in the resiliency context pays off if the tasks are of reasonable workload. We hence solely wrap compute-heavy tasks [7].

Runtime Wrapper Realisation. Whenever a task outcome has been computed, it is buffered first before we distribute it among the replicas. This is because we use non-blocking communication and the teams run asynchronously. A team progresses its computation irrespectively of teaMPI's communication. Without 
task outcome buffering, outgoing data may become inconsistent, as the application might already overwrite the send buffer with new data. To avoid some of the overhead of the buffering, we wrap the sends into another condition clause: We check before line 9 in Algorithm 1 once more whether the task outcome has been received already. If so, we know that at least one replica is ahead and has multicasted the task outcomes to the other teams while we computed locally. There is no need to distribute the local outcome once more.

Buffering is also required on the receiver side. A zero-copy approach is impossible, as the receiving rank might be slightly ahead of the sender and compute the task already itself while it receives data. Alternatively, it might lag behind and might not even have created the task including its output data fields.

\subsection{Implementation Pitfalls}

An efficient implementation of task migration between teams is technically delicate, as we are confronted with a highly dynamic communication pattern. We cannot predict which team will be fast or how many tasks the teams exchange. Instead, we receive unexpected MPI messages, as task outcomes arrive unexpectedly, while it is essential to fully overlap task sharing-related communication with the application and to receive shared task outcomes as quickly as possible.

MPI Progression. Even though launched through non-blocking MPI, messages may actually not be progressed internally by the MPI implementation [19]. Instead, communication request handles need to be checked for completion repeatedly through MPI_Test for example. Furthermore, standard MPI neither triggers an interrupt if messages arrive unexpectedly nor supports a mechanism to tell the runtime when investments into MPI progression calls actually pay off.

Too little investment into MPI progression would be lethal for our task sharing approach. We are dealing with unexpected messages which might use a rendez-vous protocol. If they are not detected in a timely manner, they are useless for the replicas and even might delay the baseline application. We therefore need frequent MPI_Iprobes. Probes detect (unexpected) incoming messages and thus issue MPI_Irecvs for pending incoming tasks which consequently are progressed through MPI_Test. Shared task outcomes consequently arrive timely.

Different to our previous work where we did interweave MPI progression with the standard tasking [26], we found it vital for teaMPI to dedicate one core to an asynchronously running communication thread [19], similar to our previous work $[20,25]$. It is responsible for both the progression of MPI messages (using MPI testing), MPI_Iprobes for detecting messages, as well as the progression of the task sharing algorithm (e.g., buffering received task outcomes and inserting them into the task outcome database). It ensures that task sharing actually overlaps and is hidden from the user code.

Memory and Communication Overhead. Task sharing runs risk to result in an excessive memory footprint and to yield many outstanding MPI receive and send handles. Due to the buffering, open communication requests do not allow us to 


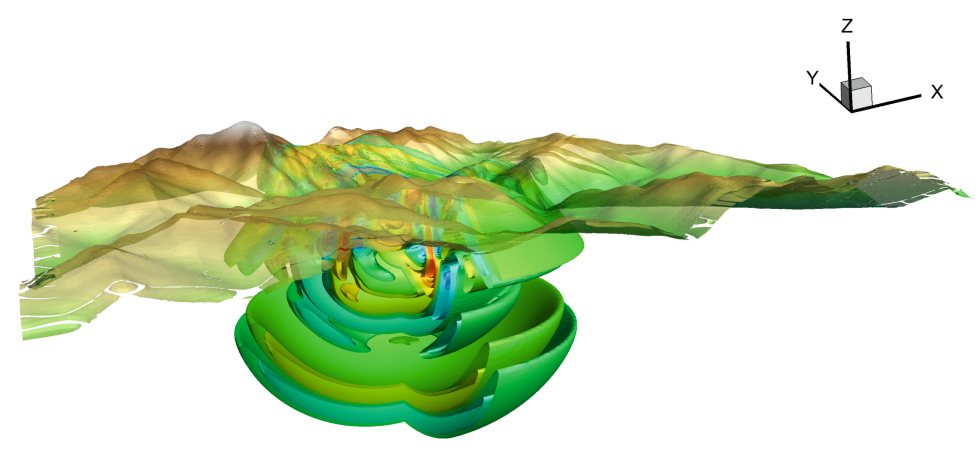

Fig. 2. Visualization of an example setup simulated with our code by courtesy of Maurizio Tavelli [30].

free allocated buffers and handles. We therefore limit the number of open send requests per process. This effectively constrains the memory overhead and also the number of open data exchange handles.

For explicit time stepping, this artificial limitation makes it convenient to drop incoming tasks immediately if they belong to a past time step. In our algorithmic blueprint, it is rare that this happens: If a rank is significantly ahead of a replica, it has fed the replica's team with task outcomes which in turn makes the replica skip all task outcome sharing. Once we limit the number of tasks, we however might run into situations where ranks receive outdated task outcomes. While the garbage collection would remove these as well, it is reasonable to pipe the incoming data into a temporary buffer right away.

\section{Results}

Our tests are conducted on the SuperMUC-NG supercomputer operated by the Leibniz Supercomputing Centre. SuperMUC-NG consists of Intel Skylake Xeon Platinum 8174 nodes, where each node hosts $2 \times 24$ cores, running at a nominal clock frequency of $2.3 \mathrm{GHz}$. Intel Omnipath serves as interconnect. We use Intel MPI, Intel Compiler 2019, and Intel's TBB for the multithreading.

We benchmark performance and functionality against a seismology simulation for the LOH.1 setup [10]. The simulation relies on an engine for solving systems of hyperbolic partial differential equations (PDEs) and employs an explicit, high order Discontinuous Galerkin scheme in space and time (ADER-DG). Its spatial discretisation stems from dynamically adaptive Cartesian meshes, while the code phrases its execution in tasks relying on TBB. Although we study a benchmark, i.e., strip the code off many features such as the integration of real geometries and subsurface data or extensive postprocessing and I/O, these core features already make up a challenging setup characterising production runs. An example visualization obtained with our framework is shown in Fig. 2. 


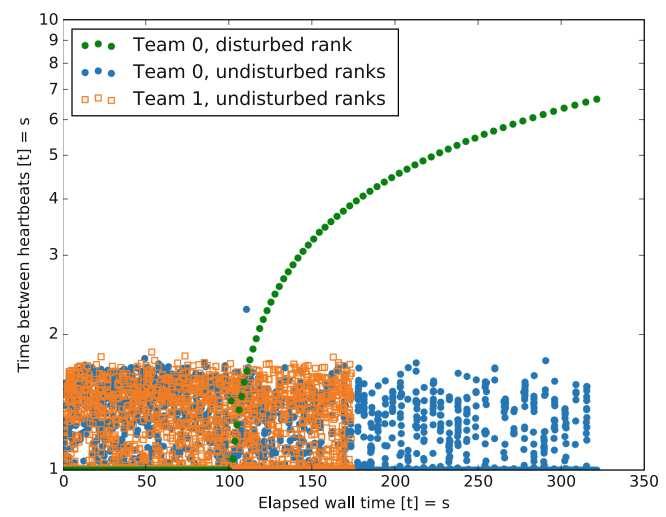

Fig. 3. Time between heartbeats on 56 nodes on SuperMUC-NG if a single node is increasingly delayed.

ADER-DG is a numerical scheme splitting up each time step into a space-time prediction, a Riemann solve and a correction phase. We found the prediction to be responsible for the majority of the runtime [6] and thus make only prediction tasks migration-ready.

One core is sacrificed to a communication thread with task sharing. If not stated differently, we do not take this additional core into account when we compare the performance of teaMPI with task sharing to a baseline code without task sharing: both the baseline as well as the task sharing versions use the same number of cores for computations. We, however, also provide data for one setup where both the baseline and the task sharing variant use the same number of cores per process: i.e., the task sharing variant uses one core less for computation than the baseline due to the core dedicated to the communication thread. Readers may recalibrate all other data accordingly or agree that the progression thread is a workaround for an MPI weakness.

\subsection{Heartbeats}

We first demonstrate how teaMPI can be used to identify failing or slow ranks with ExaHyPE. Let 56 nodes of SuperMUC-NG host two teams, each consisting of 28 MPI ranks. Each rank is responsible for one part of the three-dimensional grid. It is evenly distributed, i.e., the setup is load-balanced. We configure each rank to send a heartbeat every $\Delta t_{\mathrm{HB}}=1 \mathrm{~s}$, but artificially delay one rank in the first team in order to simulate a failing node. This is achieved by repeatedly pausing and resuming its process. A delay of $0.1 \mathrm{~s}$ kicks in after $100 \mathrm{~s}$. From here, the delays increase by $0.1 \mathrm{~s}$ every time. This resembles an anticipated scenario where a failing node gradually decreases its clock frequency before it finally goes down completely. teaMPI's goal has to be to identify this situation on time.

A plot of the time in-between heartbeats over the first 20 timesteps (Fig. 3) unmasks the failing rank. This rank delays its whole team 0. As a result, 


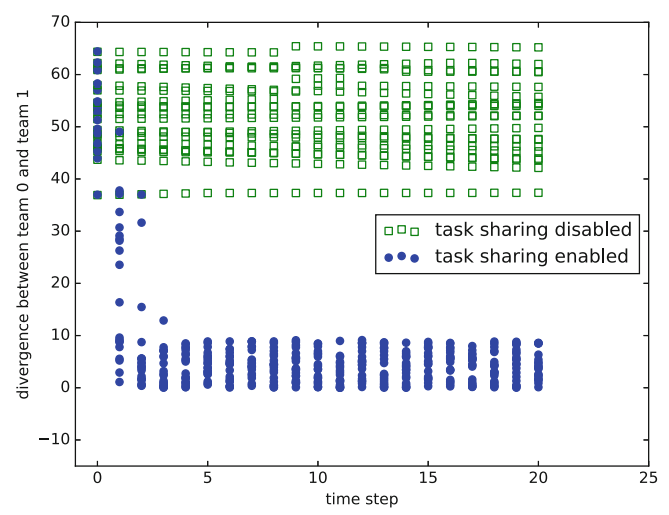

Fig. 4. Team divergence for two teams for different initial delays.

team 0 finishes later and posts more heartbeats compared to the ranks in team 1. Although one heartbeat per second is chosen, the task-based heartbeat implementation makes the actual task timings become fuzzy and consistently exceed 1 s, resulting in scheduling effects.

Every rank observes its replicas through the heartbeats. We cannot directly, i.e., in an unfiltered way, use the in-between time between heartbeats to identify failures. Instead, time averages have to be used to assess the healthiness of a replica. Although a slow rank affects all members of its team (and it is thus difficult to identify a failing rank by measuring time per time step of a team), our heartbeats are well-suited to identify which rank is to blame for a delay.

\subsection{Robustness Against Temporary Delays}

If a single rank and, hence, team is temporarily delayed through $\mathrm{I} / \mathrm{O}$ or non-persistent hardware deteriorations (overheating) for example, task sharing should enable the delayed team to "catch up" with the faster teams. To validate this hypothesis, we rerun the two-team setup but artificially delay the startup of one rank of the first team: We pause the rank for a certain time $t$ directly at startup. To exclude stochastic effects, we make $t \in[45 \mathrm{~s}, 65 \mathrm{~s}]$ uniformly distributed and run the code with and without task outcome sharing.

Let $t_{i}^{(A)}$ be the timestamp of the start of the $i$-th timestep of team $A$. For teams $A$ and $B$, we can then quantify the divergence at the $i$ th timestep as $d_{i}^{(A, B)}=\left|\left(t_{i}^{(A)}-t_{i}^{(B)}\right)\right|$. Without task sharing, an initial start offset between both teams persists throughout the simulation (Fig.4), while task outcome sharing makes the divergence decrease rather quickly: the fast team "drags along" the slow team, as it feeds it with task results.

We investigate this effect further by plotting the accumulated number of reused tasks with task sharing for the two teams (Fig. 5). Initially, the undisturbed team reuses little to no task results from the replica team, as the disturbed 


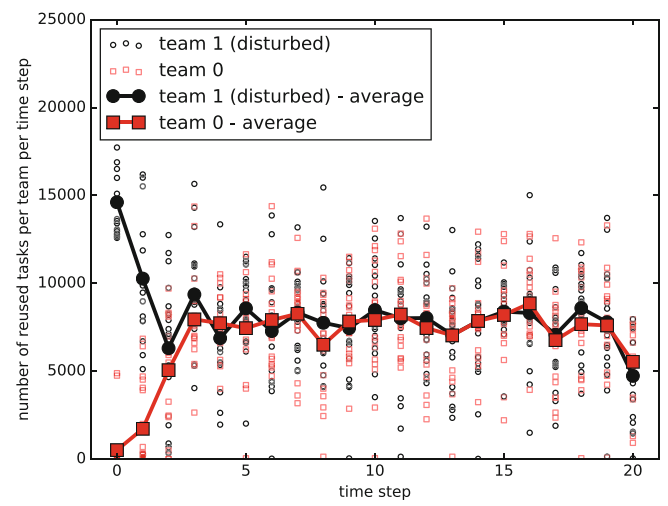

Fig. 5. Number of reused tasks per team per time step for different initial random delays (with task sharing).

team cannot provide its results in a timely manner. At the same time, the disturbed team reuses tasks starting from the first timestep. It catches up. For the delayed team, the number of reused tasks per time step decreases over time as it catches up. Accordingly, the number of reused tasks per time step increases for the undisturbed team. Once the delayed team has catched up, the teams share tasks evenly as a result of our shuffling approach.

\subsection{Upscaling}

We next study two strong scaling setups with two teams, where we gradually increase the number of cores per rank or team, respectively (Fig. 6). We compare the task sharing measurements to both a baseline that uses the same number of cores for computation (Fig. 6a) and to a baseline that uses one additional core for computation (the core that is sacrificed to a communication thread in the task sharing variant, Fig. 6b). We start with a domain decomposition of the computational grid that is well-balanced, using 28 partitions for example. These 28 partitions are mapped onto 28 ranks which are all deployed to one node. Then we grant each rank more and more cores until the experiment eventually spreads all 28 nodes. We do a similar experiment with 731 ranks or partitions, respectively. This setup eventually employs 731 nodes and 35088 cores. Each experiment is conducted for three polynomial orders of the underlying Discontinuous Galerkin scheme. The polynomial order determines how expensive the compute-heavy tasks for which we enable task sharing are relative to the total runtime. The higher the order the more dominant these tasks.

Task sharing yields a speedup of up to $1.5 \times$ for most measurements. In fact, task sharing can even compete with a baseline that uses an additional core for computation in some cases, although at reduced speedups (compare Fig. 6a and Fig. 6b). However, both experiments run into strong scaling effects at higher core counts: If the number of cores per rank exceeds a certain threshold, the 


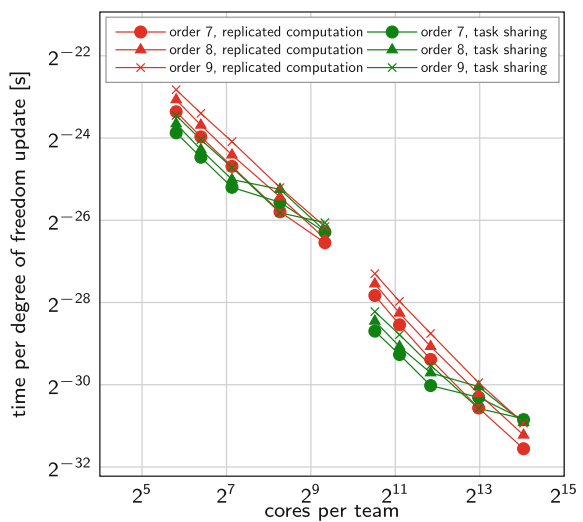

(a) Same number of computation cores for baseline and task sharing.

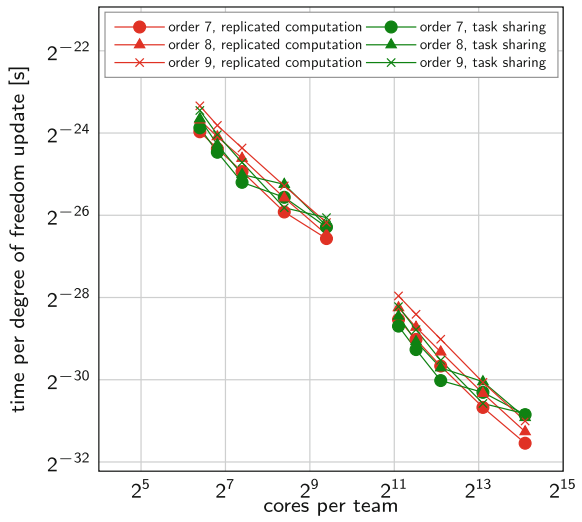

(b) Baseline uses one additional core for computation compared to task sharing.

Fig. 6. Up-scaling of two teams to up to 731 nodes and 35088 cores for varying polynomial orders: Green lines show the normalized times per degree of freedom update if task sharing is enabled, while the red lines illustrate the vanilla variant where computation is done redundantly. (Color figure online)

speedup induced by teaMPI's replication breaks down. This breakdown occurs the earlier the smaller the polynomial order, i.e., the smaller the relative cost of the shared compute tasks is relative to the total compute time. In the breakdown regime, the rate of reused task outcomes decreases significantly up to the point where hardly any computed result can be picked up by another team and all computations are effectively replicated. We invest twice the compute resources, but obtain the time-to-solution of a run without any replication.

For most setups, our task outcome sharing however pays off. Our two teams double the number of cores and thus compute cost, but we get replication plus a significant speedup by means of walltime. The advantageous property is lost if the balancing of cores per rank to compute cost of the shared tasks becomes disadvantageous - which is a direct implication of "too many cores per rank": With too many, the pressure on the communication system increases as tasks are processed and sent at a higher speed. The single communication thread and the interconnect can no longer sustain a fast enough transfer rate of task results. It just becomes cheaper to run all computations locally even though they are done somewhere else, too.

We continue our experimental section with further experiments where we use more than two teams (Table 1 ). The speedup behaviour persists, yet, we need an even higher relative compute load per task to benefit from yet another team. More than three teams does not lead to any significant improvement of the time to solution anymore. As three teams are sufficient to implement resiliency where two "valid" ranks overrule the outcome of a corrupted one, we conclude that any usage of more than three teams is likely esoteric. To confirm this hypothesis, experiments with validation routines however are required. This is out of scope here. 
Table 1. Total cost (in CPU hours) and speedup (in time-to-solution) with task sharing, each normalized to a single-team baseline at varying polynomial orders and number of cores per team.

\begin{tabular}{l|l|l|l|l|l|l|l}
\hline \multicolumn{3}{l|}{} & \multicolumn{3}{l|}{ Total cost (CPUh) } & \multicolumn{3}{l}{ Speedup (time-to-solution) } \\
\hline Order & Cores/team & 2 teams & 3 teams & 4 teams & 2 teams & 3 teams & 4 teams \\
\hline 7 & 56 & 1.39 & 1.69 & 2.22 & 1.43 & 1.77 & 1.80 \\
\hline 7 & 112 & 1.38 & 1.73 & 2.04 & 1.45 & 1.74 & 1.96 \\
\hline 7 & 224 & 1.35 & 1.66 & 1.93 & 1.48 & 1.81 & 2.07 \\
\hline 7 & 448 & 1.36 & 1.60 & 1.85 & 1.47 & 1.88 & 2.17 \\
\hline 8 & 56 & 1.35 & 1.63 & 2.05 & 1.49 & 1.84 & 1.96 \\
\hline 8 & 112 & 1.34 & 1.61 & 1.92 & 1.50 & 1.86 & 2.08 \\
\hline 8 & 224 & 1.30 & 1.57 & 1.84 & 1.54 & 1.92 & 2.18 \\
\hline 8 & 448 & 1.30 & 1.53 & 1.71 & 1.54 & 1.96 & 2.34 \\
\hline 9 & 56 & 1.30 & 1.61 & 1.94 & 1.54 & 1.86 & 2.06 \\
\hline 9 & 112 & 1.25 & 1.47 & 1.81 & 1.61 & 2.04 & 2.21 \\
\hline 9 & 224 & 1.24 & 1.44 & 1.68 & 1.62 & 2.09 & 2.38 \\
\hline 9 & 448 & 1.21 & 1.47 & 1.57 & 1.65 & 2.03 & 2.54 \\
\hline
\end{tabular}

$k$-fold replication comes at the expense of $k$-times increased total memory consumption plus increased communication needs. On top of this, the bookkeeping of task outcomes requires further resources. We quantified the memory overhead of task sharing by repeatedly sampling each rank's memory consumption during program execution (Fig. 7) after the computational grid has been allocated. In conjunction with system noise, task sharing yields a variable memory consumption pattern as task outcomes are allocated and freed dynamically. Yet, the typical additional memory overhead of task sharing remains under control at around $20 \%$ additionally used memory.

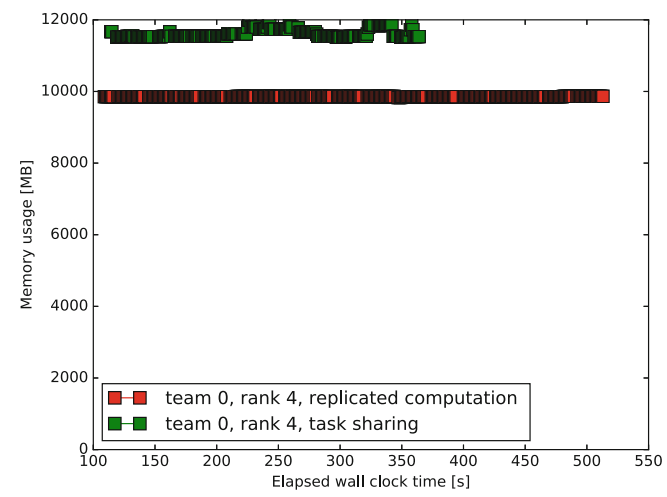

Fig. 7. Memory consumption of task sharing vs the baseline variant without task sharing for a selected representative rank. 


\section{Conclusion and Outlook}

Our paper introduces teaMPI, an MPI wrapper/plugin which replicates a simulation multiple times. We call the replicas teams. The teams run completely asynchronously. They do however exchange heartbeats. If the time in-between the heartbeats received vs. the local heartbeats diverges, we consider this to be a reliable indicator for faults. While any rank can spot any performance degradation of a replica rank, it is important to note that the heartbeats do not synchronise the replicas at all and, thus, do not introduce any performance penalty. The actual compute cost of replicas is reduced as we make each rank share its task outcomes with the replicas which, whenever these task outcomes drop in on time, skip their local computations and instead use the results from another rank from another team. This technique reduces the total CPUh cost, as long as the computation is phrased in tasks, and as long as we do not work in the strong scaling regime: Enough ready tasks have to be available, so we can shuffle their order and do not make threads idle.

Our paper introduces an elegant, minimalist and powerful new idea rendering replication in HPC economically feasible. It is however a conceptional piece of work. To translate it into a production environment, we need, on the one hand, the integration with modern MPI versions which support resiliency. On the other hand, we have to solve four further fundamental challenges: First, our code lacks a mature communication performance model for task sharing. Specifically, it would never share too many task outcomes such that the overall performance suffers. Second, the task outcome sharing makes the whole simulation more sensitive to soft faults (bit flips, e.g.) [22]: If a task yields an invalid outcome, this outcome might corrupt all other teams. There is a need to develop checksums or hash techniques that can spot such cases and veto the pollution of a run with invalid data. The heartbeat messages might be canonical candidates to carry such crossvalidation records. Third, we have to generalise our notion of shareable tasks. Our strategy relies on the fact that a code yields many shareable tasks, and that these tasks make up a significant part of the runtime. To make the concept applicable to a wider range of code characteristics, we have to develop mechanisms that can migrate and share whole task subgraphs such that more fine granular tasking benefits from our ideas, too. Finally, our approach increases the pressure on the MPI interconnects. It will be subject of future work to analyse how this pressure can be reduced. To this end, we plan to investigate whether emerging technologies such as SmartNICs can be exploited to offload the task sharing fully to the network hardware and to guarantee sufficient MPI progress.

Acknowledgements. This work received funding from the European Union's Horizon 2020 research and innovation programme under grant agreement No 671698 (ExaHyPE), and from EPSRC's Excalibur programme under grant number EP/V00154X/1 (ExaClaw). It used the facilities of the Hamilton HPC Service of Durham University. We particularly acknowledge the support of the Gauss Centre for Supercomputing e.V. (www.gauss-centre.eu) for providing computing time on the GCS 
Supercomputer SuperMUC at Leibniz Supercomputing Centre (www.lrz.de). Thanks are due to all members of the ExaHyPE consortium who made this research possible, particularly to Dominic E. Charrier for writing most of the engine code and to Leonhard Rannabauer for development of the seismic models on top of the engine. The underlying software, i.e. both ExaHyPE [21] and teaMPI, are open source (www. exahype.org and www.peano-framework.org/index.php/teampi).

\section{References}

1. Altenbernd, M., Göddeke, D.: Soft fault detection and correction for multigrid. Int. J. High Perform. Comput. Appl. 32(6), 897-912 (2018)

2. Biswas, S., de Supinski, B.R., Schulz, M., Franklin, D., Sherwood, T., Chong, F.T.: Exploiting data similarity to reduce memory footprints. In: 2011 IEEE International Parallel and Distributed Processing Symposium, pp. 152-163 (2011)

3. Bland, W., Bouteiller, A., Herault, T., Bosilca, G., Dongarra, J.: Post-failure recovery of MPI communication capability: design and rationale. Int. J. High Perform. Comput. Appl. 27(3), 244-254 (2013)

4. Cao, C., Herault, T., Bosilca, G., Dongarra, J.: Design for a soft error resilient dynamic task-based runtime. In: 2015 IEEE International Parallel and Distributed Processing Symposium, pp. 765-774 (2015)

5. Cappello, F.: Fault tolerance in petascale/ exascale systems: current knowledge, challenges and research opportunities. Int. J. High Perform. Comput. Appl. 23(3), 212-226 (2009)

6. Charrier, D.E., et al.: Studies on the energy and deep memory behaviour of a cache-oblivious, task-based hyperbolic PDE solver. Int. J. High Perform. Comp. Appl. 33(5), 973-986 (2019)

7. Charrier, D., Hazelwood, B., Weinzierl, T.: Enclave tasking for discontinuous Galerkin methods on dynamically adaptive meshes. SIAM J. Sci. Comput. 42(3), C69-C96 (2020)

8. Chen, Z., et al: Fault tolerant high performance computing by a coding approach. In: Proceedings of 10th ACM SIGPLAN Symposium on Principles and Practice of Parallel Programming, pp. 213-223. ACM (2005)

9. Chung, J., et al.: Containment domains: a scalable, efficient, and flexible resilience scheme for exascale systems. In: SC 2012: Proceedings of the International Conference for HPC, Networking, Storage and Analysis, pp. 1-11 (2012)

10. Day, S.M., et al.: Tests of 3D elastodynamics codes: final report for lifelines program task 1A02. Technical report (2003)

11. Dongarra, J., et al.: Applied mathematics research for exascale computing. Technical report, Lawrence Livermore National Lab (2014)

12. Engelmann, C., Ong, H.H., Scott, S.L.: The case for modular redundancy in largescale high performance computing systems. In: Proceedings of 8th IASTED International Conference on Parallel and Distributed Computing and Networks, vol. 1, pp. 189-194 (2009)

13. Engelmann, C.: Scaling to a million cores and beyond: using light-weight simulation to understand the challenges ahead on the road to exascale. Future Gener. Comput. Syst. 30, 59-65 (2014)

14. Fagg, G.E., et al.: Process fault tolerance: semantics, design and applications for high performance computing. Int. J. High Perform. Comput. Appl. 19(4), 465-477 $(2005)$ 
15. Ferreira, K., et al.: Evaluating the viability of process replication reliability for exascale systems. In: 2011 International Conference for HPC, Networking, Storage and Analysis (SC), pp. 1-12 (2011)

16. Fiala, D., Mueller, F., Engelmann, C., Ferreira, K., Brightwell, R., Riesen, R.: Detection and correction of silent data corruption for large-scale high-performance computing. In: Proceedings of 25th IEEE/ACM International Conference on HPC, Networking, Storage and Analysis, pp. 78:1-78:12. ACM, November 2012

17. Göddeke, D., Altenbernd, M., Ribbrock, D.: Fault-tolerant finite-element multigrid algorithms with hierarchically compressed asynchronous checkpointing. Parallel Comput. 49(C), 117-135 (2015)

18. Heene, M., Hinojosa, A.P., Bungartz, H.-J., Pflüger, D.: A massively-parallel, faulttolerant solver for high-dimensional PDEs. In: Desprez, F., et al. (eds.) Euro-Par 2016. LNCS, vol. 10104, pp. 635-647. Springer, Cham (2017). https://doi.org/10. 1007/978-3-319-58943-5_51

19. Hoefler, T., Lumsdaine, A.: Message progression in parallel computing - to thread or not to thread? In: IEEE International Conference on Cluster Computing, pp. 213-222 (2008)

20. Klinkenberg, J., Samfass, P., Bader, M., Terboven, C., Müller, M.S.: Chameleon: reactive load balancing for hybrid MPI+OpenMP task-parallel applications. J. Parallel Distr. Comput. 138, 55-64 (2020)

21. Reinarz, A., et al.: ExaHyPE: an engine for parallel dynamically adaptive simulations of wave problems. Comput. Phys. Commun., 107251 (2020)

22. Reinarz, A., Gallard, J.M., Bader, M.: Influence of a-posteriori subcell limiting on fault frequency in higher-order DG schemes. In: IEEE/ACM 8th Workshop on Fault Tolerance for HPC at eXtreme Scale, FTXS@SC 2018, pp. 79-86 (2018)

23. Rezaei, A., Khetawat, H., Patil, O., Mueller, F., Hargrove, P., Roman, E.: End-toend resilience for HPC applications. In: Weiland, M., Juckeland, G., Trinitis, C., Sadayappan, P. (eds.) ISC High Performance 2019. LNCS, vol. 11501, pp. 271-290. Springer, Cham (2019). https://doi.org/10.1007/978-3-030-20656-7_14

24. Riesen, R., Ferreira, K., Stearley, J.: See applications run and throughput jump: the case for redundant computing in HPC. In: Proceedings of International Conference on Dependable Systems and Networks, pp. 29-34 (2010)

25. Samfass, P., Klinkenberg, J., Bader, M.: Hybrid MPI+OpenMP reactive work stealing in distributed memory in the PDE framework sam(oa $)^{2}$. In: IEEE International Conference on Cluster Computing, pp. 337-347, September 2018

26. Samfass, P., Weinzierl, T., Charrier, D.E., Bader, M.: Lightweight task offloading exploiting MPI wait times for parallel adaptive mesh refinement. In: Concurrency and Computation: Practice and Experience (2020, to appear)

27. Schroeder, B., Gibson, G.A.: A large-scale study of failures in high-performance computing systems. IEEE Trans. Depend. Secur. Comput. 7(04), 337-350 (2010)

28. Simon, T., Dorband, J.: Improving application resilience through probabilistic task replication. In: ACM Workshop on Algorithmic and Application Error Resilience, June 2013

29. Subasi, O., Yalcin, G., Zyulkyarov, F., Unsal, O., Labarta, J.: Designing and modelling selective replication for fault-tolerant HPC applications. In: 17th IEEE/ACM International Symposium on Cluster, Cloud and Grid Computing (CCGRID), pp. 452-457 (2017)

30. Tavelli, M., Dumbser, M., Charrier, D.E., Rannabauer, L., Weinzierl, T., Bader, M.: A simple diffuse interface approach on adaptive Cartesian grids for the linear elastic wave equations with complex topography. J. Comput. Phys. 386, 158-189 (2019) 